\title{
CARACTERÍSTICAS ORGANIZACIONAIS E A UTILIZAÇÃO DA GESTÃO DE CUSTOS NO PROCESSO DECISÓRIO
}

\section{ORGANIZATIONAL CHARACTERISTICS AND THE USAGE OF COSTS MANAGEMENT IN DECISION-MAKING PROCESS}

\author{
ANTONIO ZANIN \\ Universidade Comunitária da Região de Chapecó. Endereço: Rua Eurico \\ Gaspar Dutra, 859E, | São Cristóvão | 89803201 | Chapecó/SC | Brasil. \\ (1) http://orcid.org/0000-0001-7837-7375 \\ zanin@unochapeco.edu.br
}

\section{CRISTIAN BAÚ DAL MAGRO}

Universidade Comunitária da Região de Chapecó. Endereço: Avenida Coronel Ernesto Francisco Bertaso, 966, | Centro | 89850-000 | Quilombo/SC | Brasil. Dhttp://orcid.org/0000-0002-7609-5806

crisbau@unochapeco.edu.br

\author{
SADY MAZZIONI \\ Universidade Comunitária da Região de Chapecó. Endereço: Rua Francisco \\ Norberto Bonher, número 55E, | Jardim Itália | 89802-530 | Chapecó/SC | \\ Brasil. \\ (1) http://orcid.org/0000-0002-8976-6699 \\ sady@unochapeco.edu.br
}

\section{RESUMO}

O objetivo do estudo é verificar os fatores determinantes das práticas de gestão de custos utilizadas no processo decisório de empresas da região Oeste catarinense. O levantamento de dados foi elaborado a partir da aplicação de questionário respondido por gestores de 41 empresas de distintas atividades. A pesquisa descritiva utilizou abordagem quantitativa com o uso dos testes quiquadrado e Kruskal-Wallis. Os resultados indicaram que o método de custeio variável é o mais utilizado pelas empresas da amostra. O ramo de atividade, forma de tributação e faturamento anual mostraram-se fatores decisivos para a utilização das ferramentas de custos de produção e orçamento. O ramo de atividade mostrou-se um fator decisivo na utilização das informações de custos para formação do preço de venda e na negociação com fornecedores e clientes. O tempo de atividade apontou diferenças sobre a utilização das informações de custos para formação do preço de venda e controle dos gastos. A forma de tributação e o número de funcionários mostraram-se decisivos para a utilização das informações de custos que atendam à legislação fiscal e também na identificação de possíveis gargalos no processo produtivo e nos preços praticados.

Palavras-chave: Características organizacionais. Gestão de custos. Práticas de custos. Ferramentas de custos. 


\begin{abstract}
The objective of this study is to verify the determinants of costs management practices used in decision-making process of companies in the westside of Santa Catarina. The data collection was carried out using a questionnaire answered by 41 companies managers from different market sectors. The descriptive research used a quantitative approach using the Chi-Square and KruskalWallis tests. The results indicated that the variable costing method is widely used by the studied companies. The market sector, tax regime and annual turnover were decisive factors for the use of production costing and budget tools. The market sector proved to be a decisive factor in the use of costs information for the selling price formation and negotiation with suppliers and customers. The uptime pointed to differences in the use of costs information for the selling price formation and expenses controlling. The tax regime and the number of employees were decisive for the use of costs information that complies with the tax legislation, identifying possible hindrances in the productive process and also in the set prices.
\end{abstract}

Keywords: Organizational characteristics. Costs management. Costs practices. Costs tools.

\title{
1 INTRODUÇÃO
}

A gestão de uma empresa, independentemente do ramo de atividade, necessita utilizar informações de custos para participar de um mercado cada vez mais competitivo. Kim, Han, Yi e Chang (2016) observam que, para o crescimento de um empreendimento, faz-se necessário que a administração se empenhe na adequada gestão de custos.

As organizações devem utilizar os métodos de custeio como importante instrumento para o processo de gestão, proporcionando informações fundamentais para a tomada de decisões (Zanievicz, Beuren, Santos \& Kloppel, 2013), dentre as quais, aquelas que servem de base para formar o preço de venda. Sob a ótica da entidade, quanto maior o preço, maior o lucro e melhor o resultado. No entanto, é preciso estar atento ao mercado, pois os limites de preços são definidos pelo mercado consumidor e pelo valor percebido e atribuído ao produto e/ou serviço comercializado (Bruni \& Famá, 2012; Nakamura \& Steinsson, 2011).

Nesse sentido, as empresas de menor porte não têm dado a devida atenção à gestão de custos e a formação do preço de venda (Heidhues \& Kőszegi, 2014). Essa conduta gera interferências gerenciais, pois, além do preço de venda, muitas outras decisões são tomadas a partir de custos, tais como: o que e quanto produzir; volume mínimo de vendas; orçamento; e gestão de custos (Martins, 2011; Afonso, Wernke \& Zanin, 2018; Souza \& Diehl, 2009).

Abbas, Gonçalves e Leoncine (2012) consideram que a utilidade da contabilidade de custos não difere entre empresas de tamanhos distintos, uma vez que desempenha as mesmas funções básicas de sistematização e análise dos gastos, classificação e contabilização dos custos, geração de relatórios e informações sobre custos de produção. Por sua vez, Callado e Pinho (2015) relatam que as práticas de gestão de custos têm sido associadas à natureza das características operacionais de atividade econômica e de porte da qual as empresas fazem parte.

Zuccolotto e Colodeti Filho (2007) consideram que muitas empresas de pequeno porte não evoluíram, inclusive, em seus modelos de decisões gerenciais, apresentando apenas um controle individual do proprietário. Além disso, Mazo (2003) relata que as empresas menores apresentam característica estrutural e organizacional geradora de problemas comuns, dos quais se destacam a deficiência do sistema de informação e a dependência do envolvimento dos dirigentes em todas as decisões da empresa.

Callado e Pinho (2015) reforçam os dispositivos que beneficiam a atuação das empresas de menor porte, como é o caso do regime de tributação no Simples Nacional. Esse tratamento tem por finalidade incentivá-las, uma vez que, em geral, as pequenas empresas, no início de suas 
atividades, não dispõem de recursos financeiros e tecnológicos suficientes, o que tem contribuído para a fragilidade nos sistemas de gestão e controle.

Nesse contexto, a questão norteadora do estudo é: quais os fatores que podem determinar as práticas de gestão de custos no processo decisório de empresas da região Oeste de Santa Catarina? O objetivo é verificar tais fatores.

A justificativa do estudo está atrelada à importância das informações produzidas na gestão de custos, diante da concorrência acirrada entre as empresas em um mercado mundial, no qual a pressão pela redução de custos é fundamental para a sobrevivência. Contribui-se em oferecer explicações das características empresariais que determinam os formatos de gestão de custos nas empresas, oferecendo subsídios para que os interessados entendam os motivos que geram deficiências nessa gestão das organizações.

\section{GESTÃO DE CUSTOS E MÉTODOS DE CUSTEIO}

A pressão por colocar no mercado produtos com qualidade e preços competitivos tem sido o principal foco dos gestores de empresas, independentemente do porte. A gestão de custos tem sido importante para manutenção da margem de lucro e da competividade nas empresas. Assim, as empresas precisam fazer esforços para gerir e reduzir os custos, bem como definir estratégias de precificação de produtos/serviços (Motta \& Escrivão Filho, 2002; Beuren \& Schlindwein, 2008).

Um controle efetivo dos custos no processo de um produto/serviço extrapola as determinações contábeis e pode ser útil no auxílio gerencial (Bornia, 2010; Chapko, Perkins, Fortney \& Maciejewski, 2009). Além desse benefício, tal controle também poderá proporcionar ao gestor a apuração do custo dos produtos como um dos critérios para fixação de preços e para analisar a rentabilidade das atividades e produtos da empresa (Callado, Miranda \& Callado, 2003; Gonzalez, Nachtmann \& Pohl, 2017).

Antes de definir o método de custeio, torna-se necessário efetuar a classificação dos custos, que de acordo com a forma de alocação, podem ser diretos e indiretos. Bruni \& Famá (2012) definem os diretos como sendo os que se consegue quantificar e alocar diretamente ao produto, sem margens de erro. Já os custos indiretos são os que precisam de bases ou taxas para sua alocação, pois são comuns a diversos produtos (Zanievicz et al., 2013).

Outra classificação importante ocorre em função da variação no volume de produção, sendo fixos ou variáveis. Os custos fixos são aqueles que não variam em função do volume de produção (a exemplo do aluguel e dos salários), enquanto os variáveis mudam de forma diretamente proporcional ao volume de produção, a exemplo das matérias-primas e materiais de embalagem (Souza \& Clemente, 2011; Lauscher \& Beuren, 2004).

Após a apuração dos custos e sua devida classificação, é necessário definir o método de custeio, dentre os quais citam-se: a) custeio por absorção: os custos indiretos são alocados aos produtos pelo volume de produção, horas máquinas, mão de obra direta e matérias-primas; b) custeio variável: os custos indiretos não são levados à análise de resultados dos produtos, estes passam a ser avaliados pelas margens de contribuição; c) custeio baseado em atividades: permite às empresas estimar os custos de fabricação e também os custos aos diferentes consumidores; d) custeio baseado em atividade e tempo (TDABC): simplifica a aplicação do custeio baseado em atividades e utiliza o tempo como principal direcionador de custo aos produtos; e) método das unidades de esforço de produção: modelo de cálculo da produção do período por meio da determinação de uma unidade de medida comum aos produtos e processos; d) Custo padrão: seu objetivo é o de fixar uma base de comparação entre o que ocorreu de custo e o que deveria ter ocorrido (Rizzi \& Zanin, 2018; Kaplan \& Anderson, 2007; Aillón, Rocha \& Marques, 2018). Portanto, para a definição do método de custeio ideal, deve ser levado em conta o tipo de informações que os gestores esperam e o seu custo de implantação (Afonso et al., 2018; Hoozée \& Hansen, 2017; Markantonis, Meyer \& Schwarze, 2012). 


\section{DECISÕES COM BASE EM CUSTOS}

As tomadas de decisões baseadas em custos são inúmeras, dentre as quais destacam-se: formação do preço de venda; análise do custo $\times$ volume $\times$ lucro; margem de contribuição dos produtos; ponto de equilíbrio; margem de segurança. Portanto, estabelecer o preço de venda com base em custos é crucial para a continuidade dos negócios, independentemente do ramo e do porte das organizações (Dalci, Tanis \& Kosan, 2010; Heidhues \& Köszegi, 2014).

A formulação dos preços está ligada às condições de mercado, exigências governamentais, custos, nível de atividade e remuneração do capital investido. O cálculo do preço de venda deve levar a um valor que traga a maximização dos lucros para o negócio; que seja possível manter a qualidade, atendendo aos anseios do mercado e aproveitar, de maneira otimizada, os níveis de produção ou serviços (Souza \& Diehl, 2009; Nakamura \& Steinsson (2011); Rizzi \& Zanin, 2018).

Santos (2012) argumenta que, para formar o preço de venda, utiliza-se de distintos métodos: método do custo de mercadorias, que leva em consideração o custo das mercadorias, produtos ou serviços; método baseado nas empresas concorrentes, o qual acompanha o preço de mercado; método baseado nas características de mercado, destacando-se a qualidade mínima do produto, questões culturais etc.; e o método misto, que parte do custo da mercadoria, verifica os preços praticados pelo mercado e o mercado que se pretende atender.

Outras informações baseadas em custos são utilizadas pelas empresas para tomada de decisões, dentre as quais destaca-se o ponto de equilíbrio. Para tanto, inicia-se pelo cálculo da margem de contribuição, que é a diferença entre o preço de venda e os custos e despesas variáveis (Martins, 2011; Bruni \& Famá, 2012). A margem de contribuição contribui para o ranqueamento dos produtos, proporcionando aos gestores definir os produtos que devem ser priorizados no sentido de melhorar o resultado (Santos, 2012; Machado \& Souza, 2006).

O ponto de equilíbrio contábil é o valor mínimo que a empresa precisa vender para equilibrar a receita com os custos e despesas totais (Martins, 2011), ou seja, a empresa terá resultados positivos a partir do ponto de equilíbrio, podendo fazer promoções e/ou vendas em outras regiões com margens reduzidas. O ponto de equilíbrio econômico, além de cobrir toda a estrutura de custos e despesas, busca definir o valor mínimo de receitas para cobrir a margem de lucro desejada pelos acionistas/proprietários de empresas (Bruni \& Famá, 2012).

Diante do contex to teórico apresentado, vislumbra-se a complexidade existente para uma organização estabelecer uma gestão de custos eficiente ao processo decisório, devendo-se considerar também fatores intrínsecos às organizações, como: ramo de atividade, regime de tributação, tempo na atividade e tamanho da empresa pelo número de empregados e faturamento.

\section{METODOLOGIA}

O estudo tem característica de pesquisa descritiva, com coleta de dados por meio de levantamento, com análise dos dados de forma quantitativa. A população da pesquisa é composta por 208 clientes de um escritório de contabilidade na região Oeste de Santa Catarina.

A coleta dos dados ocorreu por meio da utilização de questionário, instrumento cientificamente desenvolvido e utilizado para medir características importantes de indivíduos e de empresas (Hair, Babin, Money \& Samouel, 2005), o qual foi enviado aos 208 clientes do escritório, retornando 41 respostas (19,7\% da população), constituindo a amostra da pesquisa. O questionário foi desenvolvido conforme a percepção dos autores sobre os instrumentos de custos utilizados no processo decisório das organizações.

A Tabela 1 mostra o constructo das variáveis categóricas dos fatores organizacionais e dos métodos de custeio utilizados pela empresa na tomada das decisões. 
Tabela 1

\section{Constructo das variáveis categóricas dos fatores organizacionais e métodos de custeio utilizados pela empresa}

\begin{tabular}{|c|c|c|}
\hline \multicolumn{2}{|c|}{ Variáveis } & Mensuração \\
\hline \multirow{5}{*}{ Fatores organizacionais } & Ramo de atividade & Indústria; Comércio; Serviços; Transporte \\
\hline & Tempo de atividade & $\begin{array}{l}\text { Até } 3 \text { anos; de } 4 \text { a } 8 \text { anos; de } 9 \text { a } 15 \text { anos; de } 16 \text { a } 30 \text { anos; } \\
\text { acima de } 30 \text { anos. }\end{array}$ \\
\hline & Regime de tributação & Simples nacional; Lucro real; Lucro presumido. \\
\hline & Número de funcionários & Até $25 ;$ de 26 a 50; de 51 a $75 ;$ de 76 a 100; acima de 100. \\
\hline & Faturamento anual em $\mathrm{R} \$$ & $\begin{array}{l}\text { Até } 180.000,00 ; \text { de } 180.000,01 \text { a } 360.000,00 ; \text { de } 360.000,01 \\
\text { a } 1.200 .000,00 ; \text { de } 1.200 .000,01 \text { a } 4.800 .000,00 ; \text { acima de } \\
4.800 .000,01 \text {. }\end{array}$ \\
\hline \multicolumn{2}{|c|}{ Método de custeio utilizado pela empresa } & $\begin{array}{l}\text { Absorção; variável; Custeio Baseado em Atividades (ABC); } \\
\text { custeio baseado em atividade e tempo (TDABC); Unidade } \\
\text { de Esforço de Produção (UEP); custo padrão; } \\
\text { Outro(s): especifique; não utiliza. }\end{array}$ \\
\hline
\end{tabular}

Fonte: elaborado pelos autores.

Conforme a Tabela 1, foram determinadas cinco variáveis de mensuração dos fatores organizacionais: ramo de atividade, tempo de atividade, regime de tributação, número de funcionários e faturamento anual em $\mathrm{R} \$$. Por fim, tem-se a variável de averiguação do método de custeio utilizado pela empresa na tomada de decisão.

Destaca-se que todas as variáveis expostas na Tabela 1 são categóricas e, portanto, para seu devido tratamento estatístico, que busca determinar a associação entre os fatores organizacionais e o método de custeio da empresa, utilizou-se o teste qui-quadrado. Na sequência, a Tabela 2 demonstra as variáveis de mensuração das práticas de gestão de custos pelas empresas pesquisadas.

Tabela 2

\section{Constructo das variáveis em escala das práticas de gestão de custos}

\begin{tabular}{|c|c|c|c|}
\hline Variável & Afirmação & Escala & Sigla \\
\hline $\begin{array}{c}\text { Realidade da } \\
\text { empresa sobre as } \\
\text { práticas de } \\
\text { gestão de custos }\end{array}$ & $\begin{array}{l}\text { A empresa utiliza método de custeio para a formação do preço de venda. } \\
\text { A empresa está satisfeita com o método de custeio empregado na formação do } \\
\text { preço de venda. } \\
\text { A empresa utiliza software integrado para o cálculo dos custos e para } \\
\text { operacionalizar o método de custeio. } \\
\text { Existe, na empresa, algum tipo de controle interno que auxilie a gestão, como } \\
\text { planilhas, anotações etc. } \\
\text { Na apuração de custos, a empresa considera a segregação entre custos fixos e } \\
\text { variáveis. } \\
\text { Na apuração de custos, a empresa considera a segregação entre custos diretos e } \\
\text { indiretos. } \\
\text { A empresa apura os custos por departamentos. } \\
\text { Os custos indiretos são alocados aos produtos. } \\
\text { A empresa determina metas de custos (custo padrão) para os produtos como } \\
\text { forma de controle. } \\
\text { A empresa analisa as variações de materiais diretos e de mão de obra direta. } \\
\text { A empresa analisa as variações dos custos indiretos de produção. } \\
\text { O controle de custos está atrelado ao orçamento ou ao planejamento estratégico } \\
\text { da empresa. } \\
\text { A empresa utiliza-se do custo do produto para a formação do seu preço de } \\
\text { venda. } \\
\text { O estabelecimento do preço de venda condiz com as necessidades para que se } \\
\text { alcance o retorno dos investimentos. } \\
\text { A empresa está satisfeita com o preço estabelecido do(s) seu(s) } \\
\text { produto(s)/serviço(s). } \\
\text { O preço de venda dos produtos tem boa aceitação pelos seus clientes, ou seja, } \\
\text { seu preco de venda torna sua empresa competitiva perante a concorrência. }\end{array}$ & $\begin{array}{c}\text { Likert de } 5 \text { pontos } \\
\text { (1-discordo totalmente; } \\
\text { e } 5 \text { - concordo } \\
\text { totalmente })\end{array}$ & $\begin{array}{l}\text { RAE1 } \\
\text { RAE2 } \\
\text { RAE3 } \\
\text { RAE4 } \\
\text { RAE5 } \\
\text { RAE6 } \\
\text { RAE7 } \\
\text { RAE8 } \\
\text { RAE9 } \\
\text { RAE10 } \\
\text { RAE11 } \\
\text { RAE12 } \\
\text { RAE13 } \\
\text { RAE14 } \\
\text { RAE15 } \\
\text { RAE16 }\end{array}$ \\
\hline $\begin{array}{l}\text { Hipótese para o } \\
\text { preço de venda } \\
\text { ser superior ao } \\
\text { de mercado }\end{array}$ & $\begin{array}{l}\text { Revisa os custos. } \\
\text { Utiliza estratégias/parcerias/alianças para reduzir custos de distribuição, } \\
\text { propaganda, embalagens etc. } \\
\text { Revê a cadeia de valores. } \\
\text { Reduz a margem de lucro. } \\
\text { Simplesmente acompanha o preço da concorrência. }\end{array}$ & & $\begin{array}{l}\text { PDPV1 } \\
\text { PDPV2 } \\
\text { PDPV3 } \\
\text { PDPV4 } \\
\text { PDPV5 }\end{array}$ \\
\hline
\end{tabular}




\begin{tabular}{|c|c|c|c|}
\hline $\begin{array}{l}\text { Utilização das } \\
\text { ferramentas }\end{array}$ & $\begin{array}{l}\text { Utilização dos indicadores de rentabilidade e de margem de contribuição por } \\
\text { produtos/serviços ou linhas de produtos/serviços, no intuito de auxiliar a } \\
\text { tomada de decisão. } \\
\text { Utilização dos custos de produção para a tomada de decisão. } \\
\text { Utilização de orçamentos para a tomada de decisão. } \\
\text { Utilização do custo estimado (custo padrão) para a tomada de decisão. }\end{array}$ & Likert de 5 pontos $(1-$ & $\begin{array}{l}\text { UFC } 1 \\
\text { UFC2 } \\
\text { UFC3 } \\
\text { UFC4 }\end{array}$ \\
\hline $\begin{array}{l}\text { Objetivo da } \\
\text { informaçãa de } \\
\text { custos }\end{array}$ & $\begin{array}{l}\text { Para atendimento da legislação fiscal. } \\
\text { Para formação do preço de venda. } \\
\text { Para negociação com fornecedores elou clientes. } \\
\text { Para controle dos gastos. } \\
\text { Para identificar possíveis gargalos nos processos produtivos e preços } \\
\text { praticados. }\end{array}$ & $\begin{array}{c}\text { não utiliza; e } 5 \text { - sempre } \\
\text { utiliza). }\end{array}$ & $\begin{array}{l}\text { OBIC1 } \\
\text { OBIC2 } \\
\text { OBIC3 } \\
\text { OBIC4 } \\
\text { OBIC5 }\end{array}$ \\
\hline
\end{tabular}

Fonte: elaborado pelos autores.

Verifica-se na Tabela 2 a existência de quatro variáveis para a mensuração das práticas de gestão de custos, sendo que para a análise dos dados de averiguação da associação entre as variáveis categóricas e ordinais foi utilizado o teste Kruskal-Wallis. Para a aplicação dos testes estatísticos utilizou-se o software SPSS ${ }^{\circledR}$.

\section{RESULTADOS E DISCUSSÕES}

Apresenta-se, inicialmente, a estatística descritiva dos fatores organizacionais e do método de custeio utilizado no processo decisório das empresas estudadas, conforme exposto na Tabela 3.

Tabela 3

Estatística descritiva dos fatores organizacionais e método de custeio no processo decisório

\begin{tabular}{|c|c|c|c|c|c|}
\hline \multicolumn{6}{|c|}{ Fatores Organizacionais } \\
\hline Ramo de atividade & Indústria & Comércio & Serviço & Tran & nsporte \\
\hline Frequência & 21 & 13 & 3 & & 4 \\
\hline Porcentagem & $51,2 \%$ & $31,7 \%$ & $7,3 \%$ & &, $8 \%$ \\
\hline Tempo de atividade & Até 3 anos & 4 a 8 anos & 9 a 15 anos & 16 a 30 anos & Acima de 30 anos \\
\hline Frequência & 3 & 10 & 6 & 17 & 5 \\
\hline Porcentagem & $7,3 \%$ & $24,4 \%$ & $14,6 \%$ & $41,5 \%$ & $12,2 \%$ \\
\hline Regime de tributação & \multicolumn{2}{|c|}{ Simples nacional } & Lucro real & \multicolumn{2}{|c|}{ Lucro presumido } \\
\hline $\begin{array}{l}\text { Frequência } \\
\text { Porcentagem }\end{array}$ & \multicolumn{2}{|c|}{$\begin{array}{c}16 \\
39 \%\end{array}$} & $\begin{array}{c}15 \\
36.6 \%\end{array}$ & \multicolumn{2}{|r|}{$\begin{array}{c}10 \\
24,4 \%\end{array}$} \\
\hline $\mathrm{N}^{\circ}$ de funcionários & Até 25 & De 26 a 50 & De 51 a 75 & De 76 a 100 & Acima de 100 \\
\hline Frequência & 27 & 9 & 0 & 2 & 3 \\
\hline Porcentagem & $65,9 \%$ & $22 \%$ & $0,0 \%$ & $4,9 \%$ & $7,3 \%$ \\
\hline $\begin{array}{l}\text { Faturamento anual R\$ } \\
\text { (milhões) }\end{array}$ & 180.000 & $\begin{array}{c}\text { De } 180.000 \text { a } \\
360.000 \\
\end{array}$ & $\begin{array}{c}\text { De 360.000 a } \\
1.200 .000 \\
\end{array}$ & $\begin{array}{c}1.200 .000 \mathrm{a} \\
4.800 .000\end{array}$ & $\begin{array}{l}\text { Acima de } \\
4.800 .000 \\
\end{array}$ \\
\hline Frequência & 1 & 3 & 8 & 14 & 15 \\
\hline Porcentagem & $2,4 \%$ & $7,3 \%$ & $19,5 \%$ & $34,2 \%$ & $36,6 \%$ \\
\hline \multicolumn{6}{|c|}{ Método de custeio utilizado no processo decisório } \\
\hline Método de Custeio & Absorção & Variável & Custo Padrão & $\begin{array}{l}\text { ABC-TDABC- } \\
\text { UEP }\end{array}$ & Não utiliza \\
\hline Frequência & 11 & 15 & 6 & 0 & 9 \\
\hline Porcentagem & $26,8 \%$ & $36,6 \%$ & $14,6 \%$ & $0,0 \%$ & $22 \%$ \\
\hline
\end{tabular}

Fonte: dados da pesquisa.

Verifica-se, na Tabela 3, maior concentração de empresas dos ramos industrial e comercial. No tempo de atividade, as empresas estão distribuídas proporcionalmente, sendo possível apontar maior presença de empresas que atuam há mais tempo no mercado (mais de 16 anos). Quanto ao regime de tributação, observa-se distribuição homogênea entre os enquadramentos tributários, o que pode oferecer resultados satisfatórios entre a associação dos regimes tributários com as práticas de gestão de custos. Com relação ao tamanho das empresas, pelo número de funcionários e faturamento anual, considera-se a existência de concentração no nível empregatício de 50 funcionários e, por outro lado, uma parcela relevante de empresas com faturamento anual acima de $\mathrm{R} \$ 1.200 .000,00$. 
Por fim, os achados indicam que as empresas utilizam, em maior proporção, o método de custeio absorção e custeio variável na tomada de decisão. Por outro lado, observa-se um número relevante de empresas que não utilizam quaisquer métodos de custeio no suporte ao processo decisório. Além disso, as empresas pesquisadas não têm utilizado os métodos de custeio que exigem maior demanda profissional, comprometimento, softwares avançados, sistemas integrados, como é o caso do ABC, TDABC e UEP.

Os achados corroboram com Kaplan \& Anderson (2007) e Van Der Merwe (2009), os quais identificaram que muitas empresas têm dificuldades na implantação e manutenção do ABC. Além disso, semelhante ao identificado no contexto brasileiro, especificamente na região Sul, Zhou (2013) relata que na China também existe baixa adesão ao uso do ABC na gestão de custos. Sugere-se que os achados podem ser explicados pelo alto custo de implementação e pela complexidade no seu processo, fatores que têm inibido as empresas de menor porte.

Além disso, Aillón et al. (2018) fazem algumas críticas ao ABC, ao apontarem que nenhuma empresa estudada pelos autores conseguiu alcançar o estágio de total institucionalização do método de custeio ABC. Ao avaliarem os fatores de abandono do ABC, o pressuposto da pressão social foi o mais relevante, por não trazer inovação informacional e ser custoso para implementação.

Como análise complementar às práticas de gestão de custos, tem-se na Tabela 4 a estatística descritiva das práticas de gestão de custos no processo decisório organizacional.

Tabela 4

Estatística descritiva das práticas de gestão de custos no processo decisório

\begin{tabular}{|c|c|c|c|c|c|}
\hline Variável & Média & Desvio padrão & Variável & Média & Desvio padrão \\
\hline \multicolumn{3}{|c|}{$\begin{array}{l}\text { Questões da realidade da empresa sobre as } \\
\text { práticas de gestão de custos }\end{array}$} & \multicolumn{3}{|c|}{ Questões de utilização das ferramentas de custos } \\
\hline RAE1 & 4,073 & 1,3673 & UFC1 & 3,902 & 1,2411 \\
\hline RAE2 & 3,756 & 1,2606 & UFC2 & 3,000 & 1,7029 \\
\hline RAE3 & 2,683 & 1,7527 & UFC3 & 3,463 & 1,5984 \\
\hline RAE4 & 4,634 & 0,7986 & UFC4 & 3,659 & 1,6219 \\
\hline RAE5 & 4,439 & 1,2855 & \multicolumn{3}{|c|}{ Questões dos objetivos das informações de custos } \\
\hline RAE6 & 3,268 & 1,7033 & OBIC1 & 3,317 & 1,5722 \\
\hline RAE7 & 2,415 & 1,7026 & OBIC2 & 4,585 & 0,9213 \\
\hline RAE8 & 2,756 & 1,8409 & OBIC3 & 4,146 & 1,2760 \\
\hline RAE9 & 2,854 & 1,5900 & OBIC4 & 4,171 & 1,0701 \\
\hline RAE10 & 3,049 & 1,7022 & OBIC5 & 2,488 & 1,6143 \\
\hline RAE11 & 2,512 & 1,4684 & \multicolumn{3}{|c|}{ Questões do processo decisório } \\
\hline RAE12 & 3,220 & 1,3877 & PDPV1 & 3,976 & 1,1065 \\
\hline RAE13 & 4,463 & 1,0271 & PDPV2 & 3,390 & 1,3394 \\
\hline RAE14 & 3,951 & 1,0943 & PDPV3 & 2,878 & 1,5032 \\
\hline RAE15 & 3,756 & 0,9945 & PDPV4 & 3,415 & 1,1827 \\
\hline RAE16 & 4,244 & 0,7342 & PDPV5 & 3,268 & 1,5170 \\
\hline
\end{tabular}

Nota: $\mathrm{N}=41 ;$ Mínimo $=1,0 ;$ Máximo = 5,0.

Fonte: dados da pesquisa.

Observa-se, na Tabela 4, que as empresas utilizam algum método de custeio (RAE1) e de controle dos custos de produção (RAE13) para formação do preço de venda, contemplando ainda, a segregação dos custos diretos e indiretos (RAE5). A maioria das empresas utilizam os controles internos (RAE4), fazendo com que o preço seja aceito pelos clientes (RAE16).

Por outro lado, as evidências apontam que as empresas têm apresentado baixa preocupação com a utilização de software integrado para cálculo dos custos e operacionalização dos métodos de custeio (RAE3), com a apuração dos custos por departamento (RAE7), bem como a análise das variações dos custos indiretos de produção. Esses fatores são importantes para o controle eficiente dos custos, sendo primordial para o método de custeio adotado e no estabelecimento da tomada de decisão consistente e segura. 
Com relação às ferramentas de custos, os achados indicam a utilização da rentabilidade e margem de contribuição por produtos/serviços ou linhas de produtos/serviços (UFC1), bem como o custo estimado (UFC4). Contudo, o custo de produção tem sido o item menos utilizado como ferramenta de custos (UFC2). A baixa utilização do custo de produção pode ser explicada pela alta incidência de utilização desse tipo de controle nas empresas industriais, contudo, parte das empresas pesquisadas pertencem a outros ramos de atividade.

As empresas indicaram que o objetivo da informação de custos é pautado na formação do preço de venda (OBIC2), negociação com fornecedores/clientes (OBIC3) e para controle de gastos (OBIC4). Por outro lado, os gargalos nos processos produtivos e preços praticados apresentaram menor utilidade para as empresas estudadas (OBIC5). Nesse sentido, faz-se necessário estimular as empresas sobre a importância da análise dos gargalos no processo produtivo, tendo em vista que esse fator pode influenciar no aumento dos custos e, consequentemente, no preço de venda praticado e na margem de lucro estabelecida pela empresa.

Por fim, destaca-se que as empresas revisam seus custos (PDPV1), utilizam estratégias de alianças para distribuição, propaganda e embalagens (PDPV2) e acompanham o preço da concorrência (PDPV3), na hipótese de o preço de venda ser superior ao mercado. Conclui-se que as empresas têm estabelecido estratégias para fazer com que o preço de venda torne-se acessível e competitivo ao mercado, tendo em vista que a revisão dos custos pode demonstrar aspectos de desperdícios importantes; a estratégia de aliança pode trazer ganhos de barganha; e o acompanhamento no preço da concorrência pode trazer discussões que fazem com que os gestores façam adaptações, mantendo a qualidade e reduzindo custos.

Diante do cenário apresentado, torna-se relevante verificar a associação entre os fatores organizacionais e a utilização de determinados métodos de custeio, bem como delinear sobre as diferentes práticas de gestão de custos utilizadas no processo decisório.

$\mathrm{Na}$ Tabela 5, demonstra-se os fatores organizacionais que explicam a escolha por determinado método de custo, sendo utilizado o teste qui-quadrado.

Tabela 5

Teste qui-quadrado entre os fatores organizacionais e os métodos de custeio utilizados no processo decisório

\begin{tabular}{|c|c|c|c|c|c|c|c|c|}
\hline \multirow{2}{*}{\multicolumn{2}{|c|}{ Fatores organizacionais }} & \multirow{2}{*}{ Contagem } & \multicolumn{6}{|c|}{ Métodos de custeio no processo decisório } \\
\hline & & & 1 & 2 & 3 & 4 & Total & Sig. \\
\hline \multirow{8}{*}{$\begin{array}{l}\text { Ramos de } \\
\text { atividade }\end{array}$} & \multirow{2}{*}{ Indústria } & Observada & 9 & 3 & 5 & 4 & 21 & \multirow{8}{*}{$0,005^{*}$} \\
\hline & & Esperada & 5,6 & 7,7 & 3,1 & 4,6 & 21 & \\
\hline & \multirow{2}{*}{ Comércio } & Observada & 0 & 10 & 0 & 3 & 13 & \\
\hline & & Esperada & 3,4 & 4,8 & 1,9 & 2,9 & 13 & \\
\hline & \multirow{2}{*}{ Serviço } & Observada & 0 & 0 & 1 & 2 & 3 & \\
\hline & & Esperada & 0,8 & 1,1 & 0,4 & 0,7 & 3 & \\
\hline & \multirow{2}{*}{ Transporte } & Observada & 2 & 1 & 0 & 1 & 4 & \\
\hline & & Esperada & 1,0 & 1,5 & 0,6 & 0,9 & 4 & \\
\hline \multirow{6}{*}{$\begin{array}{l}\text { Regime de } \\
\text { tributação }\end{array}$} & \multirow{2}{*}{ Simples nacional } & Observada & 1 & 7 & 2 & 6 & 16 & \multirow{6}{*}{$0,064 * *$} \\
\hline & & Esperada & 4,3 & 5,9 & 2,3 & 3,5 & 16 & \\
\hline & \multirow{2}{*}{ Lucro real } & Observada & 6 & 3 & 4 & 2 & 15 & \\
\hline & & Esperada & 4,0 & 5,5 & 2,2 & 3,3 & 15 & \\
\hline & \multirow{2}{*}{ Lucro presumido } & Observada & 4 & 5 & 0 & 1 & 10 & \\
\hline & & Esperada & 2,7 & 3,7 & 1,4 & 2,2 & 10 & \\
\hline \multirow{10}{*}{$\begin{array}{l}\text { Número de } \\
\text { funcionários }\end{array}$} & \multirow{2}{*}{ Até 25} & Observada & 5 & 11 & 3 & 8 & 27 & \multirow{8}{*}{$0,036^{*}$} \\
\hline & & Esperada & 7,2 & 9,9 & 4,0 & 5,9 & 27 & \\
\hline & \multirow{4}{*}{ De 76 a 100} & Observada & 4 & 3 & 1 & 1 & 9 & \\
\hline & & Esperada & 2,4 & 3,3 & 1,3 & 2 & 9 & \\
\hline & & Observada & 0 & 0 & 2 & 0 & 2 & \\
\hline & & Esperada & 0,5 & 0,7 & 0,3 & 0,5 & 2 & \\
\hline & \multirow{2}{*}{ Acima de 100} & Observada & 2 & 1 & 0 & 0 & 3 & \\
\hline & & Esperada & 0,8 & 1,1 & 0,4 & 0,7 & 3 & \\
\hline & \multirow{2}{*}{ Até $180.000,00$} & Observada & 0 & 0 & 0 & 1 & 1 & \multirow[t]{2}{*}{$0,028 *$} \\
\hline & & Esperada & 0,3 & 0,4 & 0,1 & 0,2 & 1 & \\
\hline
\end{tabular}


Características organizacionais e a utilização da gestão de custos no processo decisório

\begin{tabular}{ccccccccc}
\hline & De $180.000,01$ a & Observada & 0 & 0 & 0 & 3 & 3 & \\
& $360.000,00$ & Esperada & 0,8 & 1,1 & 0,4 & 0,7 & 3 & \\
& De $360.000,01$ a & Observada & 0 & 5 & 1 & 2 & 8 & 8 \\
Faturamento & $1.200 .000,00$ & Esperada & 2,1 & 2,9 & 1,2 & 1,8 & 8 & \\
anual em R\$ & De $1.200 .000,01$ a & Observada & 4 & 6 & 2 & 2 & 14 & \\
& $4.800 .000,00$ & Esperada & 3,8 & 5,1 & 2,0 & 3,1 & 14 \\
& Acima de & Observada & 7 & 4 & 3 & 1 & 15 \\
& $4.800 .000,01$ & Esperada & 4,0 & 5,5 & 2,2 & 3,3 & 15 \\
\hline
\end{tabular}

Legenda: Métodos de custeio: 1 - custeio absorção; 2 - custeio variável; 3 - custeio padrão; 4 - não utiliza método de custeio.

* Significância ao nível de 5\%

Fonte: dados da pesquisa.

Destaca-se que o tempo de atividade não apresentou associação, estatisticamente significativa, com os métodos de custeio utilizados no processo decisório e, portanto, essa variável não foi apresentada na Tabela 5. O número de funcionários na escala de 51 a 75 não foi incluso porque nenhuma empresa apresentou essa característica.

De maneira geral, os resultados indicam que o ramo de atividade, regime de tributação, número de funcionários e faturamento anual são determinantes do método de custeio utilizado pelas empresas. Pode-se inferir que as empresas do ramo industrial estão associadas à utilização do método de custeio absorção, as comerciais ao uso do custeio variável, e as empresas do ramo de serviços não se utilizam dos métodos de custeio.

Borgert e Silva (2005) já haviam salientado que o segmento industrial se volta, mais tradicionalmente, para a aplicação do sistema de custeio por absorção. Contudo, tal afirmação não pode ser dirigida para as empresas do ramo de serviços, confirmando os achados desta pesquisa, conforme já mencionado por Cardoso, Pereira e Guerreiro (2004). Além disso, os achados indicaram que as indústrias também têm predomínio pelo custeio padrão, corroborando os achados de Souza, Fontana e Boff (2010), os quais relataram que o custo padrão é a prática mais utilizada por indústrias de grande porte da cidade de Caxias do Sul/RS.

Em relação ao regime de tributação, destaca-se que as empresas enquadradas no simples nacional utilizam o custeio variável e/ou não utilizam nenhum método de custeio. Por outro lado, as empresas enquadradas no lucro real estão associadas à utilização do custeio absorção e, ainda, as empresas do lucro presumido utilizam o custeio variável seguido do custeio absorção. Esse fato pode ser explicado pela legislação do imposto de renda de pessoa jurídica, a qual determina que todos os custos sejam alocados aos produtos, independentemente dos diretos ou indiretos (absorção), já que estes afetam o resultado final, base para cálculo do imposto de renda de pessoa jurídica e contribuição social sobre o lucro.

Os achados indicam que as empresas menores, pelo critério de número de funcionários, estão associadas à utilização do custeio variável. Além disso, as empresas maiores apresentam tendência ao uso do custeio variável e absorção, com maior evidência para o custeio absorção, pois este atende à legislação fiscal, e o custeio variável serve de base para obter a margem de contribuição de seus produtos e tomada de decisões de curto prazo. Os resultados pelo critério de faturamento anual relevaram-se semelhantes, sendo que as empresas com menor faturamento não utilizam os métodos de custeio, mas, conforme ocorre um crescimento, a empresa fica propensa à utilização do custeio variável seguido do custeio absorção.

A Tabela 6 mostra o resultado do teste de Kruskal-Wallis, que apresenta a diferença entre a realidade da empresa nas práticas de gestão de custos e os fatores organizacionais. 
Tabela 6

Diferenças entre a realidade da empresa sobre práticas de gestão de custos e os fatores organizacionais

\begin{tabular}{lccccc}
\hline $\begin{array}{c}\text { Realidade da } \\
\text { empresa }\end{array}$ & $\begin{array}{c}\text { Ramo de } \\
\text { atividade }\end{array}$ & $\begin{array}{c}\text { Tempo de } \\
\text { atividade }\end{array}$ & Tributação & $\begin{array}{c}\text { Número de } \\
\text { funcionários }\end{array}$ & $\begin{array}{c}\text { Faturamento } \\
\text { anual }\end{array}$ \\
\hline RAE1 & $0,003^{*}$ & $0,001^{*}$ & 0,841 & 0,089 & 0,131 \\
RAE2 & $0,002^{*}$ & $0,002^{*}$ & 0,709 & 0,087 & 0,062 \\
RAE3 & 0,131 & $0,026^{*}$ & 0,106 & 0,315 & $0,019^{*}$ \\
RAE4 & 0,185 & 0,280 & 0,167 & 0,503 & 0,216 \\
RAE5 & $0,046^{*}$ & 0,254 & 0,149 & 0,215 & $0,007^{*}$ \\
RAE6 & $0,050^{*}$ & $0,013^{*}$ & $0,011^{*}$ & 0,114 & $0,003^{*}$ \\
RAE7 & 0,723 & 0,155 & $0,000^{*}$ & $0,018^{*}$ & $0,000^{*}$ \\
RAE8 & 0,207 & 0,160 & $0,000^{*}$ & 0,090 & $0,004^{*}$ \\
RAE9 & 0,097 & 0,147 & 0,137 & $0,014^{*}$ & $0,024^{*}$ \\
RAE10 & $0,005^{*}$ & $0,008^{*}$ & 0,146 & $0,000^{*}$ & $0,002^{*}$ \\
RAE11 & 0,085 & 0,490 & 0,196 & 0,110 & 0,268 \\
RAE12 & 0,556 & $0,036^{*}$ & 0,148 & 0,569 & 0,663 \\
RAE13 & $0,001^{*}$ & 0,273 & 0,273 & 0,674 & 0,870 \\
RAE14 & $0,001^{*}$ & 0,170 & 0,954 & 0,528 & 0,203 \\
RAE15 & 0,235 & 0,062 & 0,987 & 0,975 & 0,060 \\
RAE16 & $0,008^{*}$ & 0,553 & 0,691 & 0,712 & 0,782 \\
\hline
\end{tabular}

Nota. * Significância ao nível de $5 \%$.

Fonte: dados da pesquisa.

Verifica-se, na Tabela 6, que o ramo de atividade mostrou-se associado aos seguintes aspectos: utilização do método de custeio para formação do preço de venda; satisfação sobre o método de custeio utilizado na formação do preço de venda; segregação dos custos fixos e variáveis e também dos custos diretos e indiretos; análise das variações de materiais diretos e mão de obra direta; utilização do custo do produto na formação do preço de venda, com o estabelecimento do preço de venda condizente com as necessidades de alcance do retorno dos investimentos; e preço de venda com boa aceitação pelos clientes.

O tempo de atividade apresentou associação com a utilização do método de custeio para a formação do preço de venda; satisfação da empresa frente ao método de custeio utilizado; utilização de software integrado para o cálculo dos custos; segregação dos custos fixos e variáveis e também dos custos diretos e indiretos; análise das variações de materiais diretos e mão de obra direta; e controle de custos atrelado ao orçamento ou planejamento estratégico.

O regime de tributação apresentou-se associado com a apuração dos custos pela segregação entre custos diretos e indiretos; apuração dos custos por departamentos; e a alocação dos custos indiretos aos produtos. Já o número de funcionários apresentou associação com a apuração dos custos por departamentos; determinação de metas de custos aos produtos; e a análise das variações de materiais diretos e mão de obra direta.

Por fim, o faturamento anual apresentou associação com a utilização de software integrado para o cálculo dos custos; apuração dos custos considerando a segregação entre custos fixos e variáveis e também entre custos diretos e indiretos; apuração dos custos por departamentos, com custos indiretos alocados aos produtos; determinação de metas de custos para os produtos e com a análise das variações de materiais e mão de obra direta.

Nesse sentido, visando demonstrar os fatores determinantes da realidade da empresa frente às práticas de gestão de custos, tem-se o teste Kruskal-Wallis na Tabela 7. 
Tabela 7

Teste de Kruskal-Wallis dos fatores organizacionais com as práticas de gestão de custos

\begin{tabular}{|c|c|c|c|c|c|c|c|c|}
\hline \multirow{2}{*}{ Ramo de atividade } & \multicolumn{8}{|c|}{ Realidade da empresa sobre as práticas de gestão de custos } \\
\hline & RAE1 & RAE2 & RAE5 & RAE6 & RAE10 & RAE13 & RAE14 & RAE15 \\
\hline Indústria & 23,69 & 25,81 & 22,98 & 23,57 & 26,57 & 22,93 & 23,10 & 23,76 \\
\hline Comércio & 23,62 & 20,50 & 19,12 & 16,77 & 17,73 & 25,19 & 25,73 & 20,38 \\
\hline Serviços & 7,50 & 9,50 & 10,00 & 10,17 & 10,17 & 10,00 & 7,00 & 16,50 \\
\hline Transporte & 8,50 & 6,00 & 25,00 & 29,38 & 10,50 & 5,50 & 5,13 & 11,88 \\
\hline Tempo de atividade & RAE1 & \multicolumn{2}{|c|}{ RAE2 } & RAE3 & RAE6 & \multicolumn{2}{|c|}{ RAE10 } & RAE12 \\
\hline Até 3 anos & 15,67 & \multicolumn{2}{|c|}{11,50} & 19,33 & 15,83 & \multicolumn{2}{|c|}{15,50} & 16,17 \\
\hline De 4 a 8 anos & 15,65 & \multicolumn{2}{|c|}{16,70} & 16,55 & 22,75 & \multicolumn{2}{|c|}{16,80} & 29,10 \\
\hline De 9 a 15 anos & 9,58 & \multirow{2}{*}{\multicolumn{2}{|c|}{$\begin{array}{l}10,08 \\
2671\end{array}$}} & 11,33 & 7,25 & \multicolumn{2}{|c|}{10,83} & 11,00 \\
\hline De 16 a 30 anos & 26,79 & & & 26,56 & 23,91 & \multicolumn{2}{|c|}{25,03} & 20,15 \\
\hline Acima de 30 anos & 28,90 & \multicolumn{2}{|c|}{$\begin{array}{l}26,71 \\
29,00\end{array}$} & 23,60 & 27,20 & & ,20 & 22,60 \\
\hline Tributação & \multicolumn{3}{|c|}{ RAE6 } & \multicolumn{2}{|r|}{ RAE7 } & \multicolumn{3}{|c|}{ RAE8 } \\
\hline Simples nacional & \multirow{2}{*}{\multicolumn{3}{|c|}{14,47}} & \multicolumn{2}{|r|}{15,72} & \multicolumn{3}{|c|}{16,59} \\
\hline Lucro real & \multirow{2}{*}{\multicolumn{3}{|c|}{27,13}} & \multirow{2}{*}{\multicolumn{2}{|c|}{$\begin{array}{l}30,43 \\
15,30\end{array}$}} & \multicolumn{3}{|c|}{30,63} \\
\hline Lucro presumido & \multirow{2}{*}{\multicolumn{3}{|c|}{$\begin{array}{l}22,25 \\
\text { RAE7 }\end{array}$}} & & & \multirow{2}{*}{\multicolumn{3}{|c|}{$\frac{13,60}{\text { RAE10 }}$}} \\
\hline Número de funcionários & & & & \multicolumn{2}{|r|}{ RAE9 } & & & \\
\hline Até 25 & \multicolumn{3}{|c|}{18,04} & \multicolumn{2}{|r|}{17,72} & \multicolumn{3}{|c|}{16,09} \\
\hline De 26 a 50 & \multicolumn{3}{|c|}{23,67} & \multicolumn{2}{|r|}{25,00} & \multicolumn{3}{|c|}{31,17} \\
\hline De 51 a 75 & & 25,00 & & & 22,50 & & 20, & \\
\hline De 76 a 100 & & 37,00 & & & 37,50 & & 35,0 & \\
\hline Acima de 100 & Sem & respon & & Sem & respondente & & Sem resp & ndente \\
\hline Faturamento anual em R\$ & RAE3 & & AE5 & RAE6 & RAE7 & RAE8 & RAE9 & RAE10 \\
\hline Até 180.000 & 35,50 & & 2,50 & 33,00 & 37,00 & 35,00 & 37,50 & 35,00 \\
\hline De $180.000,01$ a 360.000 & 9,50 & & 1,17 & 6,00 & 11,50 & 18,67 & 22,00 & 16,67 \\
\hline De $360.000,01$ a 1.200 .000 & 16,94 & & 7,19 & 14,00 & 15,25 & 15,06 & 14,00 & 12,25 \\
\hline De $1.200 .000,01$ a 4.800 .000 & 18,50 & & 23,75 & 20,61 & 16,61 & 15,96 & 17,36 & 17,25 \\
\hline Acima de $4.800 .000,01$ & 26,83 & & 23,67 & 27,30 & 29,00 & 28,40 & 26,80 & 29,10 \\
\hline
\end{tabular}

Fonte: dados da pesquisa.

Primeiramente, com relação ao ramo de atividade, a Tabela 7 demonstra que a indústria utiliza o método de custeio para formação do preço de venda (RAE1), estando satisfeita com relação ao método empregado (RAE2). O ramo de transporte tem segregado os custos em fixos e variáveis (RAE5), e, na segregação, entre custos diretos e indiretos (RAE6), pois é um setor que difere das indústrias que possuem vários centros de custos no processo de produção. Assim, o transporte possui o setor de gestão administrativa e de armazéns, contudo, seu maior custo está vinculado ao próprio transporte, com predominância de custos variáveis.

Os achados indicam que o ramo comercial está relacionado à utilização do custo do produto na formação do preço de venda (RAE13), sendo ele condizente com as necessidades para o alcance do retorno sobre investimentos (RAE15). Uma das possíveis explicações é a de que as empresas do ramo comercial, geralmente, tendem a se preocupar com o custo de aquisição dos produtos, dando menor ênfase aos custos indiretos e fixos. Por fim, a satisfação com o preço estabelecido para os produtos/serviços segue a escala de preferência do ramo industrial, comercial, serviços e transportes.

Destaca-se que as empresas com maior tempo de atividade estão associadas à utilização (RAE1) e satisfação (RAE2) do método de custeio na formação do preço de venda. Complementarmente, as empresas com maior tempo de atividade estão associadas à utilização de software integrado para o cálculo dos custos (RAE3), segregando os custos entre diretos e indiretos (RAE6) e análise de variações de materiais e mão de obra direta (RAE10).

Por fim, os achados indicam que as empresas com tempo de atividade entre 4 e 8 anos estão associadas ao controle de custos atrelado ao orçamento e/ou planejamento estratégico. Nesse quesito, infere-se que o tempo de atividade não tem sido fator determinante para a utilização de controle de custos atrelado ao orçamento e/ou planejamento estratégico, ambos vinculados à geração de informações para o processo decisório. 
No regime de tributação, os resultados apontam que as empresas enquadradas no lucro real possuem tendência para apuração dos custos pela segregação em diretos e indiretos (RAE6), apuração dos custos por departamentos (RAE7) e alocação dos custos indiretos aos produtos (RAE8). Pode-se sugerir que o enquadramento no regime de tributação pelo lucro real não tem sido determinante na utilização de software integrado e no controle dos custos por departamentos e tampouco atrelados ao orçamento. De maneira geral, conclui-se que, mesmo com a maior necessidade de as empresas enquadradas no lucro real manterem informações periódicas de custos para a apuração do lucro tributável, elas não exercem algumas práticas de custos que são importantes para o processo decisório.

Os achados reportam a reflexão de que as empresas maiores, pelo critério do número de funcionários, apresentam tendência para práticas de gestão na apuração dos custos por departamentos (RAE7), na determinação de metas de custos para os produtos (RAE9) e na análise das variações de materiais diretos e mão de obra direta (RAE10).

Por outro lado, extremos foram encontrados no tamanho da empresa através do faturamento anual, em que as menores e maiores utilizam software integrado (RAE3), apuram os custos considerando a segregação entre diretos e indiretos (RAE6), apuram custos por departamentos (RAE7), fazem a alocação dos custos indiretos (RAE8), determinam metas de custos (RAE9) e analisam as variações de materiais diretos e mão de obra direta (RAE10).

Surpreende o resultado de as empresas menores apresentarem características de gestão de custos que se aproximam das empresas de porte maior. Por fim, destaca-se que somente as empresas maiores apresentam associação com a segregação dos custos entre fixos e variáveis (RAE5). Portanto, resultados inconclusivos foram obtidos no tamanho pelo faturamento anual da empresa, como determinante das práticas de gestão de custos.

A Tabela 8 apresenta o resultado do teste de Kruskal-Wallis da diferença entre a utilização das ferramentas de custos e os fatores organizacionais.

Tabela 8

Diferenças entre a utilização das ferramentas de custos e os fatores organizacionais

\begin{tabular}{cccccc}
\hline $\begin{array}{c}\text { Utilização das } \\
\text { ferramentas de custos }\end{array}$ & $\begin{array}{c}\text { Ramo de } \\
\text { atividade }\end{array}$ & $\begin{array}{c}\text { Tempo de } \\
\text { atividade }\end{array}$ & Tributação & $\begin{array}{c}\text { Número de } \\
\text { funcionários }\end{array}$ & $\begin{array}{c}\text { Faturamento } \\
\text { anual }\end{array}$ \\
\hline UFC1 & 0,459 & 0,897 & 0,184 & 0,945 & 0,790 \\
UFC2 & $0,012^{*}$ & $0,001^{*}$ & $0,042^{*}$ & $0,001^{*}$ & $0,001^{*}$ \\
UFC3 & $0,027^{*}$ & 0,138 & $0,073^{* *}$ & 0,613 & $0,091^{* *}$ \\
UFC4 & 0,154 & $0,096^{* *}$ & 0,193 & $0,054^{* *}$ & 0,321 \\
\hline
\end{tabular}

Nota. * Significância ao nível de 5\%; ** Significância ao nível de $10 \%$.

Fonte: dados da pesquisa.

Verifica-se, na Tabela 8, que o ramo de atividade, tributação e faturamento anual apresentaram significância estatística com a utilização das ferramentas de custos de produção (UFC2) e orçamentos (UFC3). De acordo com os resultados, a utilização do custo de produção e do orçamento pode ser determinada pelo ramo de atividade, enquadramento tributário e faturamento anual das organizações. Além disso, o tempo de atividade e o número de funcionários determinam a utilização do custo de produção (UFC2) e custo estimado (UFC4). Para demonstrar os fatores organizacionais que determinam a utilização das ferramentas de custos, apresenta-se o teste Kruskal-Wallis na Tabela 9. 
Tabela 9

Teste de Kruskal-Wallis dos fatores organizacionais com a utilização das ferramentas de custos

\begin{tabular}{|c|c|c|c|c|c|}
\hline \multicolumn{6}{|c|}{ Utilização das ferramentas de custos } \\
\hline Ramos de atividade & UFC2 & UFC3 & Número de funcionários & UFC2 & UFC4 \\
\hline Indústria & 25,79 & 23,12 & Até 25 & 17,22 & 18,37 \\
\hline Comércio & 19,38 & 19,00 & De 26 a 50 & 29,11 & 26,50 \\
\hline Serviços & 8,00 & 4,50 & De 51 a 75 & 0,00 & 0,00 \\
\hline Transporte & 10,88 & 28,75 & De 76 a 100 & 13,75 & 15,25 \\
\hline Tempo de atividade & UFC2 & UFC4 & Acima de 100 & 35,50 & 32,00 \\
\hline Até 3 anos & 17,83 & 9,33 & & & \\
\hline De 4 a 8 anos & 13,70 & 21,65 & & & \\
\hline De 9 a 15 anos & 11,83 & 15,08 & & & \\
\hline De 16 a 30 anos & 25,38 & 22,35 & Faturamento anual em $\mathbf{R} \$$ & UFC2 & UFC3 \\
\hline Acima de 30 anos & 33,60 & 29,20 & Até 180.000 & 35,50 & 33,00 \\
\hline Tributação & UFC2 & UFC3 & De $180.000,01$ a 360.000 & 17,17 & 14,00 \\
\hline Simples nacional & 18,47 & 16,34 & De $360.000,01$ a 1.200 .000 & 11,69 & 13,13 \\
\hline Lucro real & 26,93 & 22,03 & De $1.200 .000,01$ a 4.800 .000 & 18,00 & 23,39 \\
\hline Lucro presumido & 16,15 & 26,90 & Acima de 4.800.000,00 & 28,57 & 23,57 \\
\hline
\end{tabular}

Fonte: dados da pesquisa.

Conclui-se que as empresas do ramo industrial utilizam os custos de produção no processo decisório. Por outro lado, o ramo de transporte, seguido pelas indústrias, possuem maior tendência de utilização no orçamento. Ademais, é possível inferir que as empresas com maior tempo de atividade no mercado utilizam o custo de produção e o custo estimado.

Com relação ao regime de tributação, as empresas enquadradas no lucro real estão associadas ao uso do custo de produção e, por outro lado, as empresas do lucro presumido utilizam o orçamento. As empresas maiores, pelo número de funcionários, utilizam o custo de produção e o custo estimado. Por outro lado, as empresas menores, pelo faturamento anual, demonstraram-se associadas ao custo de produção e orçamento. A Tabela 10 apresenta o teste Kruskal-Wallis da diferença entre as informações de custos e os fatores organizacionais.

Tabela 10

Diferenças entre os objetivos das informações de custos e os fatores organizacionais

\begin{tabular}{cccccc}
\hline $\begin{array}{c}\text { Objetivos das } \\
\text { informações de custos }\end{array}$ & $\begin{array}{c}\text { Ramo de } \\
\text { atividade }\end{array}$ & $\begin{array}{c}\text { Tempo de } \\
\text { atividade }\end{array}$ & Tributação & $\begin{array}{c}\text { Número de } \\
\text { funcionários }\end{array}$ & $\begin{array}{c}\text { Faturamento } \\
\text { anual }\end{array}$ \\
\hline OBIC1 & 0,520 & 0,163 & $0,006^{*}$ & $0,044^{*}$ & 0,103 \\
OBIC2 & $0,000^{*}$ & $0,009^{*}$ & 0,517 & 0,189 & 0,173 \\
OBIC3 & $0,050^{*}$ & 0,163 & 0,908 & 0,511 & 0,355 \\
OBIC4 & 0,116 & $0,049^{*}$ & 0,139 & 0,814 & 0,413 \\
OBIC5 & 0,333 & 0,821 & $0,047^{*}$ & $0,024^{*}$ & 0,324 \\
\hline
\end{tabular}

Nota. * Significância ao nível de 5\%.

Fonte: dados da pesquisa.

Verifica-se, na Tabela 10, que o ramo de atividade apresentou diferença estatisticamente significativa com a utilização das informações de custos para formação do preço de venda e na negociação com fornecedores e clientes. O tempo de atividade apontou diferenças sobre a utilização das informações de custos para formação do preço de venda e controle dos gastos. Com relação à tributação e ao número de funcionários, observa-se a existência de diferença estatisticamente significativa com a utilização das informações de custos para o atendimento da legislação fiscal e na identificação de gargalos nos processos produtivos e preços praticados.

$\mathrm{Na}$ Tabela 11 apresenta-se o teste Kruskal-Wallis para identificar diferenças entre os fatores organizacionais e os objetivos das informações de custos. 
Tabela 11

Teste de Kruskal-Wallis dos fatores organizacionais com os objetivos das informações de custos

\begin{tabular}{lcc|lcc}
\hline & \multicolumn{5}{c}{ Objetivos das informações de custos } \\
\hline Ramos de atividade & OBIC2 & OBIC3 & Tributação & OBIC1 & OBIC5 \\
\hline Indústria & 23,79 & 20,69 & Simples Nacional & 14,69 & 20,47 \\
Comércio & 24,12 & 23,58 & Lucro Real & 27,97 & 25,83 \\
Serviços & 9,50 & 5,50 & Lucro Presumido & 20,65 & 14,60 \\
Transporte & 4,88 & 25,88 & & & OBIC5 \\
\hline Tempo de atividade & OBIC2 & OBIC4 & $\mathbf{N}^{\mathbf{0}}$ de funcionários & 20,93 & 17,78 \\
\hline Até 3 anos & 19,50 & 10,50 & Até 25 & 14,67 & 23,44 \\
De 4 a 8 anos & 14,90 & 26,55 & De 26 a 50 & 0.00 & 0,00 \\
De 9 a 15 anos & 15,42 & 12,50 & De 51 a 75 & 29,50 & 34,75 \\
De 16 a 30 anos & 25,50 & 23,12 & De 76 a 100 & 35,00 & 33,50 \\
Acima de 30 anos & 25,50 & 19,20 & Acima de 100 &
\end{tabular}

Fonte: dados da pesquisa.

Os achados sugerem que as empresas dos ramos comercial e industrial utilizam com maior ênfase a informação de custos para formação do preço de venda e, por outro lado, as empresas dos ramos de transporte e comercial utilizam as informações de custos para negociações com clientes e fornecedores. As empresas com maior tempo de atividade utilizam-se das informações de custos para formar, adequadamente, o preço de venda, enquanto que as empresas menores as utilizam para controlar os gastos. Ademais, empresas maiores as utilizam para atendimento a legislação e para definir os gargalos nos processos e preços praticados.

$\mathrm{Na}$ forma de tributação, os resultados relevam que as empresas do lucro real utilizam as informações de custos com o objetivo de atender à legislação e na identificação de gargalos nos processos produtivos e nos preços praticados. De maneira geral, as empresas do lucro real precisam efetuar maior controle sobre os custos para a correta determinação do custo dos produtos/mercadorias vendidas, fazendo com que seus objetivos estejam associados ao atendimento da legislação. Além disso, a fiscalização é mais rígida na forma com que as empresas do lucro real fazem os controles dos custos, sendo esse formato de tributação mais exigido em termos de obrigações e controles para fornecimento de informações ao fisco.

A Tabela 12 apresenta o resultado do teste de Kruskal-Wallis, para verificar a existência de diferença estatisticamente significativa entre o processo decisório na hipótese de o preço de venda ser superior ao de mercado e aos fatores organizacionais.

Tabela 12

\begin{tabular}{|c|c|c|c|c|c|}
\hline $\begin{array}{c}\text { Processo decisório na } \\
\text { hipótese de o preço de venda } \\
\text { ser superior }\end{array}$ & $\begin{array}{l}\text { Ramo de } \\
\text { atividade }\end{array}$ & $\begin{array}{l}\text { Tempo de } \\
\text { atividade }\end{array}$ & Tributação & $\begin{array}{l}\text { Número de } \\
\text { funcionários }\end{array}$ & $\begin{array}{c}\text { Faturamento } \\
\text { anual }\end{array}$ \\
\hline PDPV1 & 0,117 & 0,582 & $0,036^{*}$ & 0,531 & 0,804 \\
\hline PDPV2 & 0,784 & 0,711 & 0,842 & 0,767 & 0,368 \\
\hline PDPV3 & 0,353 & 0,948 & 0,283 & 0,371 & 0,412 \\
\hline PDPV4 & 0,142 & $0,052^{* *}$ & 0,402 & 0,794 & 0,285 \\
\hline PDPV5 & $0,067^{*}$ & $0,082 * *$ & 0,373 & 0,818 & 0,350 \\
\hline
\end{tabular}

Nota. * Significância ao nível de 5\%; ** Significância ao nível de $10 \%$.

Fonte: dados da pesquisa.

Verifica-se, na Tabela 12, que o ramo de atividade apresentou diferença estatística com acompanhamento no preço da concorrência, na hipótese de o preço de venda ser superior ao mercado. $\mathrm{O}$ tempo de atividade demonstrou significância com a redução na margem de lucro e acompanhamento no preço da concorrência. Por fim, o regime de tributação está associado com a revisão dos custos, na hipótese de o preço de venda ser superior ao de mercado. 
Na sequência, tem-se os resultados do teste de Kruskal-Wallis, demonstrados na Tabela 13, para verificar a existência de diferenças entre os fatores organizacionais com o processo decisório na hipótese de o preço de venda ser superior ao de mercado.

Tabela 13

Teste de Kruskal-Wallis dos fatores organizacionais com o processo decisório de formação do preço

\begin{tabular}{lccc}
\hline \multicolumn{4}{c}{ Hipótese do preço de venda ser superior ao de mercado } \\
\hline \multicolumn{1}{c}{ Tempo de atividade } & & PDPV4 & PDPV5 \\
\hline Até 3 anos & 18,67 & 18,00 \\
De 4 a 8 anos & & 12,25 & 29,30 \\
De 9 a 15 anos & 28,00 & 22,83 \\
De 16 a 30 anos & & 23,65 & 16,21 \\
Acima de 30 anos & & 22,40 & 20,30 \\
\hline Ramos de atividade & PDPV5 & Tributação & PDPV1 \\
\hline Indústria & 19,71 & Simples nacional & 19,44 \\
Comércio & 17,46 & Lucro real & 17,43 \\
Serviços & 29,50 & Lucro presumido & 28,85 \\
Transporte & 32,88 & & \\
\hline
\end{tabular}

Fonte: dados da pesquisa.

Os resultados da Tabela 13 indicam que as empresas do ramo de serviços e transporte estão mais propensas ao acompanhamento do preço da concorrência na hipótese de o preço ser superior ao de mercado. Conclui-se que as empresas do ramo de serviços e transporte estão menos preocupadas com a formação do preço de venda utilizando-se das informações de custos para a tomada de decisão, tendo em vista que apenas acompanham o preço da concorrência.

Além disso, as empresas com maior tempo de atividade reduzem a margem de lucro na hipótese de o preço de venda ser acima da concorrência. As empresas com quatro a oito anos na atividade acompanham o preço da concorrência. Por fim, as empresas no regime de tributação do lucro presumido buscam a redução nos custos, na hipótese de o preço de venda ser superior ao de mercado.

\section{CONSIDERAÇÕES FINAIS}

Os achados sugerem um grande número de empresas que não utilizam métodos de custeio, tornando-se fator de preocupação pela importância que a utilização desses métodos exerce na formação do preço de venda dos produtos e para controle dos custos nos processos decisórios. Aquelas empresas que se utilizam de métodos de custeio focalizam na absorção e variável, que são os menos complexos para implementação e, portanto, apresentando um custo-benefício razoável. Conclui-se que métodos mais avançados de custeio não têm sido ferramentas utilizáveis no contexto das organizações estudadas.

Os achados indicam a utilização do controle de custos de produção na formação do preço de venda, bem como na utilização do controle interno para estabelecimento do preço de venda mais acessível aos clientes. Um fator de preocupação está vinculado ao pouco interesse das empresas na utilização de software integrado para cálculo dos custos, além da baixa relevância na análise dos gargalos, na hipótese de um preço de venda superior à concorrência. Portanto, depreende-se que as empresas apresentam preocupação em se adaptar ao mercado, contudo, tal decisão pode provocar redução nas margens de resultados, pela não observância dos fatores que podem estar associados aos custos que geram gargalos em relação aos concorrentes.

Entende-se que as empresas do ramo industrial utilizam o método de custeio absorção, enquanto que as comerciais utilizam o custeio variável, e as demais não utilizam nenhum para a gestão. Além disso, as empresas do lucro real fazem uso do método absorção vislumbrando o atendimento à legislação, a qual orienta as organizações que utilizam esse método para formação 
do custo dos produtos vendidos para base de dedução tributária. Esse achado corrobora o estudo de Pereira e Beuren (2004), os quais concluíram que as empresas com menor número de empregados utilizam o custeio absorção, que a princípio teria como finalidade atender às exigências fiscais e societárias.

Ademais, os achados sugerem que as empresas do ramo comercial utilizam o custo do produto para formação do preço de venda, sendo estas as que mais se preocupam com o custo de aquisição, dando ênfase aos indiretos e fixos. Por fim, compreende-se que o custeio absorção pode proporcionar maior satisfação no estabelecimento do preço de produtos, tendo em vista que a escala de maior preferência foi visualizada nas empresas do ramo industrial.

O ramo de serviços e transporte está preocupado com a formação do preço de venda, sendo que, para sua formação, tais empresas têm utilizado, basicamente, o acompanhamento da concorrência. Esse resultado é preocupante, pois o acompanhamento dos custos é algo bastante relevante para o estabelecimento do preço de venda. O controle de custos nos ramos de serviços e transportes é bastante simples em comparação com outros ramos, contudo, esses tipos de organizações não demonstraram preocupação quanto a tais fatores.

Conclui-se ainda, que as empresas do simples nacional e do lucro presumido utilizam o custeio variável na determinação do preço de venda. Finalmente, as empresas maiores tendem à utilização do custeio absorção, enquanto que as menores utilizam o custeio variável e, em alguns casos, não utilizam de métodos de custeio para a tomada de decisões. Esses indícios corroboram as evidências de Callado e Pinho (2015), os quais enfatizaram que as pequenas e médias empresas possuem estruturas administrativa e operacional simples, tornando possível que suas práticas de gestão de custos se assemelhem entre si, indicando haver isomorfismo mimético entre as organizações.

Complementarmente, as empresas com maior tempo de atividade utilizam software integrado para cálculo dos custos, sendo eles segregados entre diretos e indiretos, com análise de variações de materiais e mão de obra direta. Consideração deve ser feita de que as empresas mais antigas possuem maior estrutura financeira e organizacional para investimento em softwares integrados, os quais possibilitam acurácia na segregação dos custos para tomada das decisões, como pode ser vislumbrado nos achados. Enfim, considera-se que o ramo de atividade, regime de tributação, tempo de atividade e tamanho determinem as práticas de gestão de custos.

Recomenda-se que sejam realizadas pesquisas futuras com a observância dos custos para gestão e suas características organizacionais em empresas de grande porte. Além disso, a observação de outras características de comportamento dos gestores poderiam ser utilizáveis como fatores explicativos de determinada escolha na gestão de custos.

\section{REFERÊNCIAS}

Abbas, K., Gonçalves, M. N., \& Leoncine, M. (2012). Os métodos de custeio: vantagens, desvantagens e sua aplicabilidade nos diversos tipos de organizações apresentadas pela literatura. Contexto, 12(22), 145-159.

Afonso, P. S. L. P., Wernke, R., \& Zanin (2018). A. Managing the cost of unused capacity: An integrative and comparative analysis of the ABC, TABC and UEP Methods. Revista Del Instituto Internacional de Costos, 13, 150-163.

Aillón, H. S., Rocha, W., \& Marques, K. C. M. (2018). Deinstitutionalization of activity basic costing: analysis from the new institutional sociology perspective. Revista Contabilidade Vista \& Revista, 29, 101-129.

Beuren, I. M., \& Schlindwein, N. F. (2008). Uso do custeio por absorção e do sistema RKW para gerar informações gerenciais: Um estudo de caso em hospital. ABCustos, 3(2), 27-53. 
Borgert, A., \& Silva, M. Z. (2005). Método de custeio híbrido para gestão de custos em uma empresa prestadora de serviços. Anais do Congresso Brasileiro de Custos, Florianópolis, SC, Brasil, 19.

Bornia, A. C. (2010). Análise gerencial de custos: aplicação em empresas modernas (3a ed.). São Paulo: Atlas.

Bruni, A. L., \& Famá, R. (2012). Gestão de custos e formação de preços: com aplicações na calculadora HP 12C e Excel (6a ed.). São Paulo: Atlas.

Callado, A. L. C., Miranda, L. C., \& Callado, A. A. C. (2003). Fatores associados à gestão de custos: um estudo nas micro e pequenas empresas do setor de confecções. Revista Produção, 13(1), 64-75.

Callado, A. A. C., \& Pinho, M. A. B. (2015). Evidências de isomorfismo mimético sobre práticas de gestão de custos entre micro e pequenas empresas de diferentes setores de atividade. Contabilidade Vista \& Revista, 25(2), 119-137.

Cardoso, R. L., Pereira, C. A., \& Guerreiro, R. (2004). A produção acadêmica em custos no âmbito ENANPAD: uma análise entre 1998 a 2003. Anais do Encontro Anual da Associação Nacional dos Programas de Pós-Graduação em Administração, Curitiba, PR, Brasil, 28.

Chapko, M. K., Liu, C. F., Perkins, M., Li, Y. F., Fortney, J. C., \& Maciejewski, M. L. (2009). Equivalence of two healthcare costing methods: bottom-up and top-down. Health economics, 18(10), 1188-1201.

Dalci, I., Tanis, V., \& Kosan, L. (2010). Customer profitability analysis with time-driven activity based costing: A case study in a hotel. International Journal of Contemporary Hospitality Management, 22(5), 609-637.

Gonzalez, M., Nachtmann, H., \& Pohl, E. (2017). Time-driven activity-based costing for health care provider supply chains. The Engineering Economist, 62(2), 161-179.

Hair, J., Babin, B., Money, A., \& Samouel, P. (2005). Fundamentos de métodos de pesquisa em administração. Porto Alegre: Bookman.

Heidhues, P., \& Kőszegi, B. (2014). Regular prices and sales. Theoretical Economics, 9(1), 217 251.

Hoozée, S., \& Hansen, S. C. (2017). A comparison of activity-based costing and time-driven activity-based costing. Journal of Management Accounting Research, 30(1), 143-167.

Kaplan, S. R., \& Anderson, R. S. (2007). Custeio baseado em atividades e tempo. Rio de Janeiro: Elsevier.

Kim, Y. W., Han, S. H., Yi, J. S., \& Chang, S. (2016). Supply chain cost model for prefabricated building material based on time-driven activity-based costing. Canadian Journal of Civil Engineering, 43(4), 287-293. 
Lauscher, M. A., \& Beuren, I. M. (2004). Gestão estratégica de custos. Revista de Contabilidade Vista \& Revista, 15(2), 53-84.

Machado, D. G., \& Souza, M. A. (2006). Análise das relações entre a gestão de custos e a gestão do preço de venda: um estudo das práticas adotadas por empresas industriais conserveiras estabelecidas no RS. Revista Universo Contábil, 2(1), 42-60.

Markantonis, V., Meyer, V., \& Schwarze, R. (2012). Review Article" Valuating the intangible effects of natural hazards-review and analysis of the costing methods". Natural Hazards and Earth System Sciences, 12(5), 1633-1640.

Martins, E. (2011). Contabilidade de custos (10a ed.). São Paulo: Atlas.

Mazo, E. M. (2003). Benchstar: metodologia de benchmarking para análise da gestão da produção nas micro e pequenas empresas. Dissertação de Mestrado em Engenharia de produção), Curso de Pós-Graduação em Engenharia de produção, Departamento de Engenharia de produção, Universidade de Santa Catarina, Florianópolis, SC, Brasil.

Motta, F. G., \& Escrivão Filho, E. (2002). Fatores influentes na adoção de métodos de custeio em pequenas empresas: estudo multicasos. Produto \& Produção, 6(1).

Nakamura, E., \& Steinsson, J. (2011). Price setting in forward-looking customer markets. Journal of Monetary Economics, 58(3), 220-233.

Pereira, C. C., \& Beuren, I. M. (2004). Fatores determinantes na escolha do sistema de custos para dar suporte ao processo de gestão: um estudo nas grandes empresas de Santa Catarina. Revista Catarinense da Ciência Contábil, 3(9), 9-30.

Rizzi, D. I., \& Zanin, A. (2018). Estratégia de formação de preço de venda/serviço dos empreendedores incubados na recepeti. Holos (Natal. Online), 2, 111-127.

Santos, J. J. (2012). Fundamentos de custos para formação do preço e do lucro (5a ed.). São Paulo: Atlas.

Souza, A., \& Clemente, A. (2011). Gestão de custos: aplicações operacionais e estratégicas (2a ed.). São Paulo, Atlas.

Souza, M. A., \& Diehl, C. A. (2009). Gestão de custos: uma abordagem integrada entre contabilidade, engenharia e administração. São Paulo, Atlas.

Souza, M. A., Fontana, F. B., \& Boff, C. D. S. (2010). Planejamento e controle de custos: um estudo sobre as práticas adotadas por empresas industriais de Caxias do SulRS. Contabilidade Vista \& Revista, 21(2), 121-151.

Van Der Merwe, A. (2009). Debating the principles: ABC and its dominant principle of work. Cost Management, 23(5), 20-28.

Zanievicz, M., Beuren, I. M., Santos, P. S. A., \& Kloeppel, N. R. (2013). Métodos de Custeio: uma meta-análise dos artigos apresentados no Congresso Brasileiro de Custos no período de 1994 a 2010. Revista Brasileira de Gestão de Negócios, 15(49), 601-616. 
Zhou, Y. (2013). Costing methodologies and cost management practices: A survey of Chinese companies. In 2013 International Conference on Computational and Information Sciences (pp. 454-457). IEEE.

Zuccolotto, R., \& Colodeti Filho E. (2007). Gerenciamento de preços em empresas de pequeno porte por meio do custeio variável e do método de Monte Carlo. Enfoque: Reflexão Contábil, 26(3), 39-52. 